\title{
Bi-directional diffusion induced grain boundary motion with triple junctions
}

\author{
H. GARCKE ${ }^{\dagger}$ \\ Naturwissenschaftliche Fakultät I - Mathematik, Universität Regensburg, \\ 93040 Regensburg, Germany \\ AND \\ VANESSA STYLES \\ Centre for Mathematical Analysis and Its Applications, Department of Mathematics, \\ University of Sussex, Brighton, BN1 9QH, U.K.
}

[Received 30 May 2003 and in revised form 5 January 2004]

\begin{abstract}
We propose a multi-order parameter phase field system and a sharp interface model to describe bidirectional diffusion induced grain boundary motion in the presence of triple junctions. Numerical approximations of the models are presented together with some computational results.
\end{abstract}

\section{Introduction}

Diffusion of solute atoms along a grain boundary separating two crystals of different orientation induces a transverse motion of the boundary (see [4, 17, 23]). This phenomenon is called diffusion induced grain boundary motion (DIGM) and can be observed for several systems (see [17]). In [4] a phase field model has been derived to describe DIGM and several subsequent theoretical and numerical studies [6, 7, 9, 10] showed that this model is able to capture many experimentally observed features of DIGM. Furthermore, it was shown that the phase field model allows for a free boundary model in an appropriate sharp interface limit in which forced motion by mean curvature (describing the movement of the grain boundary) is coupled to a quasi-static diffusion equation for the concentration of the solute [10]. However both the phase field model and the free boundary model are only able to describe uni-directional motion of grain boundaries between two different types of grains. In experiments variable directions of motion within a single grain boundary and the occurrence of triple junctions are observed.

In [11] and [9] models capable of describing bi-directional DIGM have been developed. The former is based on a sharp interface approach allowing for forced motion by mean curvature in two directions and assuming a thin film geometry in which the solute diffusion through the film is very rapid. In [9] three different models have been proposed which all have the feature that the forcing term is non-local. All proposed models are able to describe phenomena observed in bi-directional DIGM such as the appearance of double seams.

One goal of this paper is to derive a general phase field model with the following properties:

- it describes bi-directional DIGM,

- triple junctions can be included,

†Email: harald.garcke@mathematik.uni-regensburg.de

Email: V.Styles@sussex.ac.uk 
- the evolution is formulated with the help of local quantities,

- it allows for thin as well as thick films,

- it is thermodynamically consistent in the sense that a free energy inequality is fulfilled.

We are going to derive a model with the above properties by similar ideas to the ones used in the derivation of the model by Cahn, Fife and Penrose [4]. But as opposed to their approach we will consider more than one phase field. We will use one phase field for each of the regions that can be distinguished either by orientation or by chemical composition. Assume in the specimen under consideration there are initially $N$ different orientations present. When solute diffuses in along the boundaries between these different grains the presence of the solute may induce a motion of the boundary. In the trail of the moving grain boundary, solute is deposited and the orientation of the lattice changes. The newly formed region can be distinguished from the $N$ initially present regions because solute is now present. We will therefore introduce another $N$ phase fields for the regions in which solute is present in order to distinguish them from the ones in which there is no solute (see Figure 1).

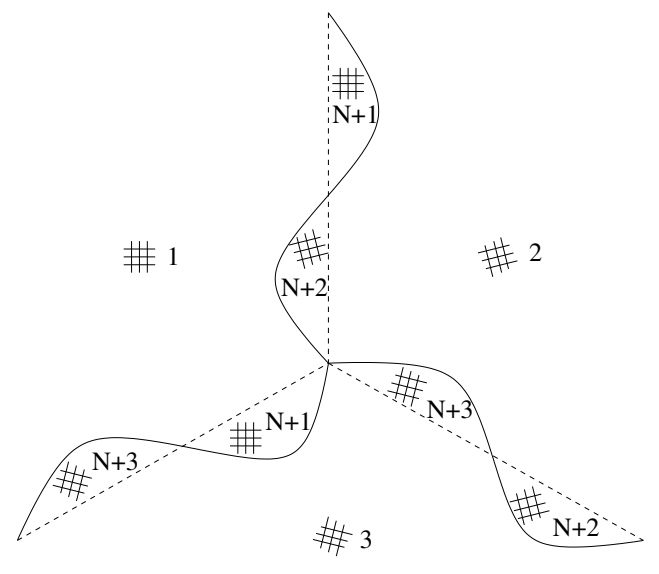

FIG. 1. DIGM at a triple junction.

In Figure 1 the dotted lines indicate the original positions of the grain boundaries. The solid lines refer to the position of the grain boundaries after some time $t>0$. The regions between the solid and dashed lines are alloyed, i.e. they contain solute.

We proceed as follows. First of all we formulate a free energy which will be a functional of the phase field vector and the concentration of the solute. The free energy will contain interfacial energy contributions, chemical energy terms and coupling terms between the phase fields and the concentration. The latter results from contributions to the free energy from elastic interactions. In the spirit of Cahn, Fife and Penrose [4] we will then derive evolution equations as a gradient flow of the free energy. Our model can be interpreted as a generalization of the model in [4] to situations with a vector phase field. But there is a major difference in the formulation of the free energy and we will discuss this issue further on.

By formally matched asymptotic expansions the phase field model can be related to a sharp interface model. In a two-dimensional setting this leads to evolution of curves that move by forced motion by mean curvature and that are coupled at triple junctions. We will discuss this model in 
Section 3 where in particular a new flux balance at triple junctions is considered. In Section 4 we formulate discretizations of both the phase field model and the free boundary model and finally in Section 5 we present numerical simulations obtained from both models.

\section{The phase field model}

We assume that in the specimen under consideration there are $N$ grain orientations present. For each of the $N$ grains we introduce a phase field $\varphi_{i}(i=1, \ldots, N)$. In addition we introduce phase fields $\varphi_{N+1}, \ldots, \varphi_{2 N}$, where $\varphi_{N+i}(i=1, \ldots, N)$ represents a grain of orientation $i$ that is alloyed (i.e. that contains solute). We note that since the orientations of the grains do not change from un-alloyed to alloyed no grain boundaries exist between grains $i$ and $i+N$. The field $\varphi_{i}$ should be interpreted as an order parameter which locally gives the fraction of the un-alloyed grain $i$ if $1 \leqslant i \leqslant N$ and the fraction of the alloyed grain $i-N$ if $N+1 \leqslant i \leqslant 2 N$. The phase space for the vector field $\varphi=\left(\varphi_{1}, \ldots, \varphi_{2 N}\right)$ is therefore defined as

$$
\Sigma:=\left\{\varphi \in \mathbb{R}^{2 N}: \sum_{i=1}^{2 N} \varphi_{i}=1\right\} .
$$

In view of this constraint, we define the projection $T$ onto the tangent space $T \Sigma:=\left\{\varphi \in \mathbb{R}^{2 N}\right.$ : $\left.\sum_{i=1}^{2 N} \varphi_{i}=0\right\}$ by

$$
(T \varphi)_{i}:=\varphi_{i}-\frac{1}{2 N} \sum_{j=1}^{2 N} \varphi_{j} .
$$

Since $\varphi_{i}$ stands for a fraction, we ask all components of the order parameter $\varphi$ to be non-negative, i.e. we want $\varphi \in \mathcal{G}$, where

$$
\mathcal{G}:=\left\{\varphi \in \Sigma: \varphi_{i} \geqslant 0\right\}
$$

is the Gibbs simplex in $\mathbb{R}^{2 N}$. Furthermore we introduce a dimensionless concentration $u$ which represents the concentration of the solute.

We study a phase field model that is based on the free energy

$$
\mathcal{E}(\varphi, u)=\int_{\Omega}\left(\frac{1}{2 \varepsilon} u^{2}+\varepsilon F(\varphi, \nabla \varphi)+\frac{1}{\varepsilon} \Psi(\varphi)+p(\varphi, u)\right),
$$

where $\Omega \subset \mathbb{R}^{d}, d=2,3$, denotes the region occupied by the specimen under consideration. The first term in the free energy takes into account the chemical energy, the second and third represent interfacial energy (cf. [19]) and the last term is an interaction term. We will always assume that $F$ is homogeneous of degree 2 in $\nabla \varphi$ and that $\Psi$ is non-negative and has minima at the corners $\mathbf{e}_{1}, \ldots, \mathbf{e}_{2 N}$ of the Gibbs simplex $\left(\mathbf{e}_{i}:=\left(\delta_{i j}\right)_{j=1, \ldots, 2 N}\right)$. Recalling that there are no grain boundaries between grains $i$ and $i+N$ we propose two gradient energy terms (normalizing the interfacial energy)

$$
F(\varphi, \nabla \varphi):=\frac{1}{2} \sum_{i=1}^{N}\left|\nabla\left(\varphi_{i}+\varphi_{i+N}\right)\right|^{2}
$$

and

$$
F(\varphi, \nabla \varphi):=\sum_{i, j=1, i<j}^{N} \sigma_{i j}\left|\varphi_{i} \nabla \varphi_{j}-\varphi_{j} \nabla \varphi_{i}\right|^{2}
$$


where in the latter case we set the surface energy $\sigma_{i j}=0$ if $j=i+N$ and for simplicity we normalize $\sigma_{i j}=1$ for all other cases. We refer to [13, 14] for more details on the second example of the gradient energy. In the former case the choice of $F(\varphi, \nabla \varphi)$ guarantees that there is no interfacial energy for a transition $i \rightarrow i+N$ because in this case it will turn out that $\varphi_{i}+\varphi_{i+N}=1$.

The interaction term $p(\varphi, u)$ is a coupling term between $\varphi$ and $u$ and takes the form

$$
p(\varphi, u)=f(u) \sum_{i=1}^{N} \varphi_{i},
$$

where $f(u)$ is a non-negative function with $f(0)=0$. We note that a more physically realistic choice for the chemical energy would be the ideal solution formula (see [4]) but for mathematical simplicity we take a quadratic function as the first term in the free energy. To simplify presentation we have set most of the material constants to be one.

The equations of motion for $\varphi$ are derived by considering the gradient flow of 2.3 giving an Allen-Cahn type equation:

$$
\begin{aligned}
\varepsilon \frac{\partial \varphi}{\partial t} & =-\frac{\partial \mathcal{E}}{\partial \varphi}(\varphi, u) \\
& =\varepsilon \nabla \cdot T F_{X}-\varepsilon T F_{\varphi}-\frac{1}{\varepsilon} T \Psi_{\varphi}(\varphi)-T p_{\varphi}(\varphi, u)
\end{aligned}
$$

together with the natural boundary condition

$$
T F_{X}(\varphi, \nabla \varphi) \cdot v=0 \quad \text { on } \partial \Omega
$$

Here $\partial \mathcal{E} / \partial \varphi$ denotes a functional derivative, $v$ is the outer unit normal to $\partial \Omega$ and the subscripts $\varphi$ and $X$ denote differentiation with respect to the variables corresponding to $\varphi$ and $\nabla \varphi$ respectively. Since the concentration $u$ obeys a conservation law we couple [2.4 with, as in [4], a kinetic equation with conserving dynamics for $u$ :

$$
\gamma \frac{\partial u}{\partial t}=\nabla \cdot\left(D(\varphi) \nabla \frac{\partial \mathcal{E}}{\partial u}(\varphi, u)\right) \quad \text { in } \Omega,
$$

which is equivalent to

$$
\gamma \varepsilon \frac{\partial u}{\partial t}=\nabla \cdot(D(\varphi) \nabla v) \quad \text { in } \Omega
$$

together with the Newton flux boundary condition

$$
D(\varphi) \frac{\partial v}{\partial v}=\alpha D(\varphi)(1-u) \quad \text { on } \partial \Omega
$$

or the Dirichlet boundary condition

$$
D(\varphi) u=D(\varphi) \quad \text { on } \partial \Omega .
$$

Here $\gamma$ is a kinetic coefficient, $\alpha$ is a large positive number and

$$
v=u+\varepsilon f^{\prime}(u) \sum_{i=1}^{N} \varphi_{i}
$$


is the scaled chemical potential. The diffusivity

$$
D(\varphi)=\prod_{i=1}^{2 N}\left(1-\varphi_{i}\right)
$$

is chosen such that diffusion is restricted to the interfacial regions, i.e. we assume that we can neglect diffusion in the bulk. If the geometry is such that the specimen under consideration is thin it can be assumed that the diffusion of solute atoms is rapid so that (2.6) is replaced by

$$
u(\mathbf{x}, t)= \begin{cases}1 & \forall(\mathbf{x}, t) \text { such that } \varphi_{i}(\mathbf{x}, \bar{t})>0 \text { for any } i \in\{N+1, \ldots, 2 N\} \text { and } \bar{t} \in[0, t], \\ 0 & \text { otherwise. }\end{cases}
$$

For $\Psi$ we take the classical obstacle potential (see [1, 14]) which is defined to be

$$
\Psi(\varphi)= \begin{cases}\sum_{i<j} \varphi_{i} \varphi_{j}=\frac{1}{2} \sum_{i=1}^{2 N} \varphi_{i}\left(1-\varphi_{i}\right) & \text { for } \varphi \in \mathcal{G} \\ \infty & \text { otherwise }\end{cases}
$$

Noting 2.1] we have

$$
\mathcal{E}(\varphi, u)=\int_{\Omega}\left(\frac{1}{2 \varepsilon} u^{2}+\varepsilon F(\varphi, \nabla \varphi)+\frac{1}{2 \varepsilon} \sum_{i=1}^{2 N} \varphi_{i}\left(1-\varphi_{i}\right)+p(\varphi, u)+I_{\mathcal{G}}(\varphi)\right),
$$

where $I_{\mathcal{G}}(\eta)$ denotes the indicator function such that

$$
I_{\mathcal{G}}(\boldsymbol{\eta})= \begin{cases}0 & \text { for } \boldsymbol{\eta} \in \mathcal{G} \\ \infty & \text { otherwise }\end{cases}
$$

With this choice of $\Psi$ we have to solve a variational inequality instead of the Allen-Cahn equation 2.4], so that a solution $\varphi$ has to satisfy

$$
\begin{aligned}
\varepsilon\left(\frac{\partial \varphi}{\partial t}, \boldsymbol{\eta}-\boldsymbol{\varphi}\right) \geqslant & -\varepsilon\left(F_{X}, \nabla(\boldsymbol{\eta}-\boldsymbol{\varphi})\right)-\left(\varepsilon F_{\boldsymbol{\varphi}}+\frac{1}{\varepsilon}\left(\frac{1}{2} \mathbb{1}-\boldsymbol{\varphi}\right), \boldsymbol{\eta}-\boldsymbol{\varphi}\right) \\
& -(f(u) \tilde{\mathbf{e}}, \boldsymbol{\eta}-\boldsymbol{\varphi})
\end{aligned}
$$

for all $\boldsymbol{\eta}: \Omega \times(0, T) \rightarrow \mathcal{G}$. Here $\mathbb{1}=\{1, \ldots, 1\} \in \mathbb{R}^{2 N}$ and $\tilde{\mathbf{e}}=\left\{\tilde{e}_{1}, \ldots, \tilde{e}_{2 N}\right\}$ with

$$
\tilde{e}_{i}= \begin{cases}1 & \text { for } i=1, \ldots, N, \\ 0 & \text { for } i=N+1, \ldots, 2 N,\end{cases}
$$

and $(\cdot, \cdot)$ denotes the standard $L^{2}$ inner product over $\Omega$. As initial data we set

$$
\varphi(\mathbf{x}, 0)=\varphi_{0}(\mathbf{x}), \quad u(\mathbf{x}, 0)=0 \quad \forall \mathbf{x} \in \Omega,
$$

which implies that initially no solute is present, but of course more general initial conditions for $u$ are possible. 


\section{A sharp interface model}

\subsection{The singular limit of the phase field model}

3.1.1 Equations at interfaces. Formal asymptotics as $\varepsilon \rightarrow 0$ similar to [2, 13] on the phase field system shows that the domain will be split into time dependent phases $\Omega_{i}$ where $\varphi$ takes the constant value $\mathbf{e}_{i}$. These domains are separated by interfaces $\Gamma$ and since the problem deals with multiple grains these interfaces may intersect at triple (or even multiple) junctions $m$.

As in the phase field model we denote the concentration of solute in $\Omega$ by $u(\mathbf{x}, t)$ for all $\mathbf{x} \in \Omega$ and we denote the concentration at an interface $\Gamma_{i}$ by $u_{i}$ so that

$$
u_{i}=u(\mathbf{x}, t) \quad \forall \mathbf{x} \in \Gamma_{i} .
$$

REMARK 3.1 It turns out that at an interface between $i$ and $i+N$ the phase fields $\varphi_{i}$ and $\varphi_{i+N}$ are discontinuous. This is due to the fact that the gradient term $F(\varphi, \nabla \varphi)$ in $\mathcal{E}(\varphi, u)$ vanishes at such an interface. Such an interface does not move because there is no term that could balance $\partial_{t} \varphi_{i}$ and $\partial_{t} \varphi_{i+N}$ in the case of movement.

Furthermore formal asymptotics in the spirit of [2, 10, 13] gives that the governing equation for an interface $\Gamma_{k}$ separating bulk phases $\Omega_{i}$ and $\Omega_{j}$ (if $i<j$ and $j \neq i+N$ ) is given by the kinetic law

$$
V_{k}=\kappa_{k}+\frac{4}{\pi} \mathcal{F}_{k}
$$

where $V_{k}$ and $\kappa_{k}$ denote the normal velocity and the curvature of the interface $\Gamma_{k}$ with respect to the normal $v_{k}$ pointing from $\Omega_{i}$ to $\Omega_{j}$ and

$$
\mathcal{F}_{k}= \begin{cases}0 & \text { if } i, j \in[1, N], \\ 0 & \text { if } i, j \in[N+1,2 N], \\ {[f(u)]_{\Gamma_{k}}} & \text { if } i \in[1, N], j \in[N+1,2 N], j \neq i+N .\end{cases}
$$

Here $[f(u)]_{\Gamma_{k}}$ denotes the jump in $f(u)$ across the interface, so that for all $\mathbf{x} \in \Gamma_{k}$,

$$
[f(u)]_{\Gamma_{k}}=\lim _{\delta \rightarrow 0}\left\{f\left(u\left(\mathbf{x}+\delta v_{k}\right)\right)-f\left(u\left(\mathbf{x}-\delta v_{k}\right)\right)\right\} .
$$

As in [10], on an interface $\Gamma_{k}$ the concentration $u_{k}$ satisfies

$$
-\gamma V_{k}[u]_{\Gamma_{k}}=\frac{\pi}{8} u_{k, s s}
$$

where $s$ denotes the arc length of $\Gamma_{k}$ and a subscript denotes differentiation with respect to arc length.

Furthermore at such grain boundaries to leading order the profile of the transition layer is a standing wave solution and we obtain

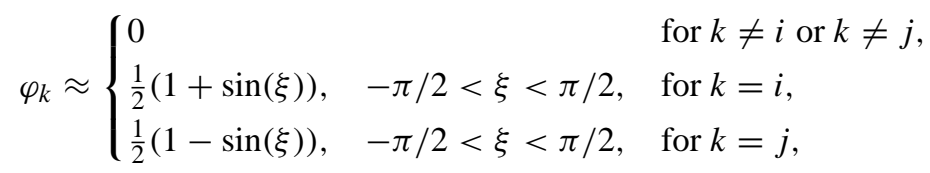

where $\xi=\varepsilon^{-1} \operatorname{dist}\left(\mathbf{x}, \Gamma_{k}(t)\right)$ with $\Gamma_{k}(t)$ denoting the interface at time $t$. 
At boundary points $B$ where an interface $\Gamma_{i}$ meets the boundary it must do so orthogonally (see [12, 21]). If the Newton flux condition (2.7) is used the concentration $u_{i}$ should satisfy

$$
u_{i, s}=\alpha\left(1-u_{i}\right) \quad \text { at } B
$$

and for the Dirichlet boundary condition 2.8 we get

$$
u_{i}=1 \quad \text { at } B \text {. }
$$

3.1.2 Triple junction conditions. In the following we derive sharp interface equations for the solute $u$ from the mass balance law and we prove free energy inequalities. We restrict ourselves to the case of a triple junction $m$ where three interfaces $\Gamma_{1}, \Gamma_{2}$ and $\Gamma_{3}$ ahead of alloyed regions $\Omega_{1}, \Omega_{2}$ and $\Omega_{3}$ respectively meet (see Figure 2). We note that the natural generalizations to more complicated geometries are possible.

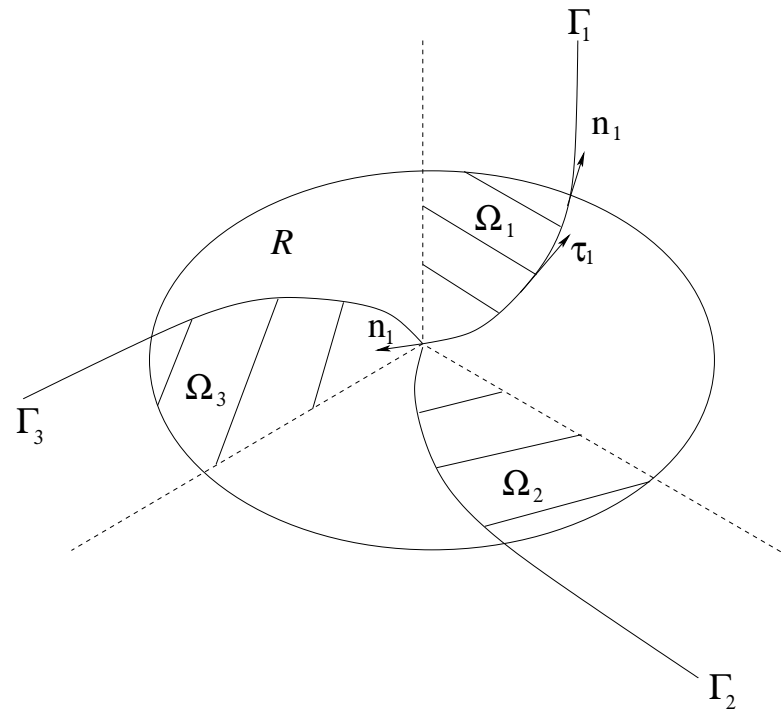

FIG. 2. Balance law

Formal asymptotics $([2,12])$ shows that the force balance

$$
\sigma_{1} \tau_{1}+\sigma_{2} \tau_{2}+\sigma_{3} \tau_{3}=0
$$

holds, where $\tau_{i}$ is the tangent to $\Gamma_{i}$ (pointing in the direction of the triple junction) and $\sigma_{i}$ is the surface energy associated with a transition across $\Gamma_{i}$. In the case that none of the interfaces separate regions of the same grain orientation all surface energies are equal and we obtain

$$
\tau_{1}+\tau_{2}+\tau_{3}=0 .
$$

REMARK 3.2 On an interface $\Gamma_{1}$ that separates regions of alloyed grain orientation $i$ and unalloyed grain orientation $i$, we assume that there is no surface energy and hence from (3.7) we conclude that

$$
\tau_{2}=-\tau_{3}
$$

thus, at such a triple junction the interfaces $\Gamma_{2}$ and $\Gamma_{3}$ join to form a $C^{1}$-curve. 
Further conditions at triple junctions have to hold for the solute concentration. The first one says that

$$
u \text { is continuous through triple junctions, }
$$

which follows from the continuity of the chemical potential (see for example [15]). The other one is

$$
\sum_{i=1}^{3} u_{i, s}=0
$$

which enforces the balance of mass for the solute. This condition can be derived by formal asymptotics similar to [15]. We will not present this here, instead in the following subsection we will derive the conditions for $u$ at the triple junction from general balance laws.

\subsection{Balance law and equilibrium conditions}

In the case of DIGM bulk diffusion can be neglected and diffusion of atoms is restricted to grain boundaries. Thus the balance of mass has the form

$$
\frac{\mathrm{d}}{\mathrm{d} t} \int_{R} u=-\sum_{i=1}^{3} \int_{\Gamma_{i} \cap \partial R} \mathbf{q}_{i} \cdot \mathbf{n}_{i}
$$

(setting $\gamma$ equal to 1 for simplicity of notation), where $R$ denotes any region in $\Omega, \mathbf{q}_{i}(\mathbf{x})$ denotes a tangential vector field which is the mass flux within the interface at a point $\mathbf{x}$, and $\mathbf{n}_{i}$ is the unit vector tangential to $\Gamma_{i}$ pointing outward to $\Gamma_{i} \cap R$ (see Figure 2). Under the constitutive assumption that

$$
\mathbf{q}_{i}=-\nabla_{\Gamma_{i}} u
$$

(setting the mass diffusivity equal to 1 ) we obtain

$$
\frac{\mathrm{d}}{\mathrm{d} t} \int_{R} u=\sum_{i=1}^{3} \int_{\Gamma_{i} \cap R} u_{i, s s}-\int_{\Gamma_{1} \cap \Gamma_{2} \cap \Gamma_{3}} \nabla_{\Gamma_{i}} u \cdot \mathbf{n}_{i} .
$$

Using a transport theorem (see [16]) one obtains (using also the fact that the concentration in front of the interface is zero)

$$
\frac{\mathrm{d}}{\mathrm{d} t} \int_{R} u=\sum_{i=1}^{3} \int_{\Gamma_{i} \cap R} V_{i} u_{i}
$$

and hence in the case that $R$ intersects only one interface we have

$$
\int_{\Gamma_{i} \cap R} V_{i} u_{i}=\int_{\Gamma_{i} \cap R} u_{i, s s} .
$$

This implies (since $R$ is arbitrary)

$$
V_{i} u_{i}=u_{i, s s} .
$$

If $R$ intersects a triple junction, using 3.13 we obtain

$$
\sum_{i=1}^{3} u_{i, s}\left(\tau_{i} \cdot \mathbf{n}_{i}\right)=0
$$

and if the curves are parametrized so that all $\tau_{i}$ point away from the triple junction we obtain 3.10 . 
REMARK 3.3 (i) The above derivation only uses the form of a mass balance given by 3.11 and the constitutive assumption (3.12) for the mass flux.

(ii) In a closed system, i.e.

$$
\mathbf{q}_{i}=0 \Leftrightarrow u_{i, s}=0 \quad \text { on } \partial \Gamma_{i} \cap \partial \Omega
$$

we obtain from 3.11

$$
\frac{\mathrm{d}}{\mathrm{d} t} \int_{\Omega} u=0
$$

(iii) Postulating a free energy of the form

$$
\sum_{i=1}^{3}\left(L_{i}(t)-\int_{\Omega_{i}} f(u)\right)
$$

where $L_{i}(t)$ is the length of $\Gamma_{i}(t)$, we can derive (3.1) in the spirit of the approach of [16], and (3.8) is the force balance at the triple junction (see [12, 18]).

The following theorem states growth properties for the free energy and also gives the existence of a Lyapunov functional in the case that the system is closed.

THEOREM 1 Let $\left(u_{1}, u_{2}, u_{3}\right)$ and $\left(\Gamma_{1}, \Gamma_{2}, \Gamma_{3}\right)$ be solutions of 3.1), 3.2. 3.4 and 3.8 -3.10 with geometry as in Figure 2 , Then

$$
\frac{\mathrm{d}}{\mathrm{d} t} \int_{\Omega} u^{2}=-\sum_{i=1}^{3} \int_{\Gamma_{i}} u_{i, s}^{2}+\sum_{i=1}^{3} \int_{\partial \Gamma_{i} \cap \partial \Omega} u_{i, s}\left(\tau_{i} \cdot \mathbf{n}_{i}\right)
$$

and

$$
\frac{\mathrm{d}}{\mathrm{d} t}\left(\sum_{i=1}^{3} L_{i}(t)-\int_{\Omega} f(u)\right)=-\sum_{i=1}^{3} \int_{\Gamma_{i}} V_{i}^{2} .
$$

For a closed system, i.e. $u_{i, s}=0$ on $\partial \Gamma_{i} \cap \partial \Omega$, we obtain

$$
\frac{\mathrm{d}}{\mathrm{d} t} \int_{\Omega} u^{2}=-\sum_{i=1}^{3} \int_{\Gamma_{i}} u_{i, s}^{2} .
$$

In the case that $f(u)=u^{2}$ we have

$$
\frac{\mathrm{d}}{\mathrm{d} t} \sum_{i=1}^{3} L_{i}(t)=-\sum_{i=1}^{3}\left(\int_{\Gamma_{i}} u_{i, s}^{2}+\int_{\Gamma_{i}} V_{i}^{2}\right) .
$$

Proof. Using [3.4, 3.10) and transport theorems (see [16]) we obtain

$$
\begin{aligned}
\frac{\mathrm{d}}{\mathrm{d} t} \int_{\Omega} u^{2} & =\frac{\mathrm{d}}{\mathrm{d} t} \int_{\bigcup_{i=1}^{3} \Omega_{i}} u^{2}=\sum_{i=1}^{3} \int_{\Gamma_{i}} u_{i}^{2} V_{i}=\sum_{i=1}^{3} \int_{\Gamma_{i}} u_{i} u_{i, s s} \\
& =-\sum_{i=1}^{3} \int_{\Gamma_{i}} u_{i, s}^{2}+\sum_{i=1}^{3} \int_{\partial \Gamma_{i} \cap \bar{\Omega}} u_{i, s}\left(\tau_{i} \cdot \mathbf{n}_{i}\right) .
\end{aligned}
$$


We denote by $\dot{e}(t)$ the velocity of an endpoint $e(t)$ of $\Gamma_{i}$. Using the fact that $\sum_{i=1}^{3} \mathbf{n}_{i}=0$ at a triple junction (see (3.8) ) and that $\dot{e}(t) \cdot \mathbf{n}_{i}=0$ at boundary points (which follows from the fact that $e(t) \in \partial \Omega$ ) we obtain

$$
\begin{aligned}
\frac{\mathrm{d}}{\mathrm{d} t}\left(\sum_{i=1}^{3} L_{i}(t)-\int_{\Omega} f(u)\right) & =\sum_{i=1}^{3}\left(-\int_{\Gamma_{i}} \kappa_{i} V_{i}+\int_{\partial \Gamma_{i}} \mathbf{n}_{i} \cdot \dot{e}(t)-\int_{\Gamma_{i}} f\left(u_{i}\right) V_{i}\right) \\
& =-\sum_{i=1}^{3} \int_{\Gamma_{i}} V_{i}\left(\kappa_{i}+f\left(u_{i}\right)\right)=-\sum_{i=1}^{3} \int_{\Gamma_{i}} V_{i}^{2}
\end{aligned}
$$

\subsection{Initial data}

Noting (3.1)-(3.4) we see that suitable initial data for the concentration need to be imposed in order for alloyed regions to nucleate. In particular to obtain a jump in concentration across an interface $\Gamma_{i}$ we need to introduce a small strip in which solute is already present. We adapt the techniques used in [9] and set

$$
u(\mathbf{x}, 0)= \begin{cases}1 & \forall \mathbf{x} \in \Lambda_{\delta} \\ 0 & \forall \mathbf{x} \in \Omega \backslash \Lambda_{\delta}\end{cases}
$$

where $\Lambda_{\delta}=\bigcup_{i=1}^{2 N} \Lambda_{i}$ and $\Lambda_{i}$ denotes a strip of width $\delta \ll 1$ lying on either the positive or the negative normal side of $\Gamma_{i}$. This gives a non-zero jump in 3.3 and hence a driving force in 3.1.

\section{Numerical discretizations}

In this section we present numerical discretizations of the phase field and sharp interface models derived in Sections 2 and 3 respectively.

\subsection{Phase field discretization}

Let $\mathcal{T}_{h}$ be a quasi-uniform triangulation of a polyhedral approximation $\Omega_{h}$ of $\Omega$ with $h:=$ $\max _{T \in \mathcal{T}_{h}} \operatorname{diam}(T)$. The finite element space $S_{h}$ is defined by

$$
S_{h}:=\left\{\chi \in C^{0}(\bar{\Omega}) \mid \chi \text { is linear on each } T \in \mathcal{T}_{h}\right\}
$$

and we set

$$
\mathcal{G}_{h}:=\left\{\boldsymbol{\eta} \in\left(S_{h}\right)^{2 N}: \sum_{i=1}^{2 N} \eta_{i}=1, \eta_{i} \geqslant 0 \forall i \in\{1, \ldots, 2 N\}\right\} .
$$

We denote by $\mathcal{N}_{h}=\left\{\mathbf{x}_{1}, \ldots, \mathbf{x}_{M}\right\}$ the set of nodes of the triangulation and by $\left\{\xi_{1}, \ldots, \xi_{M}\right\}$ the corresponding standard basis of $S_{h}$. Finally, let $\Delta t>0$ be a time step and $t_{k}=k \Delta t, k \geqslant 0$. For more details on finite element methods we refer to [5].

We discretize 2.11 using an explicit Euler scheme in time and a finite element approximation with elements in $\left(S_{h}\right)^{2 N}$ in space. Knowing the solution $\varphi_{h}^{k}$ and $u_{h}^{k}$ at time $t_{k}$ we determine $\varphi_{h}^{k+1}$ such that for all $\boldsymbol{\eta}_{h}:[0, T] \rightarrow \mathcal{G}_{h}$,

$$
\begin{aligned}
\left(\frac{\varepsilon}{\Delta t}\left(\varphi_{h}^{k+1}-\varphi_{h}^{k}\right), \boldsymbol{\eta}-\boldsymbol{\varphi}_{h}^{k+1}\right)_{h} \geqslant-\varepsilon\left(F_{X}\left(\varphi_{h}^{k}, \nabla \varphi_{h}^{k}\right), \nabla\left(\boldsymbol{\eta}-\varphi_{h}^{k+1}\right)\right)_{h} \\
-\left(\varepsilon F_{\boldsymbol{\varphi}}\left(\varphi_{h}^{k}, \nabla \varphi_{h}^{k}\right)+\frac{1}{\varepsilon}\left(\frac{1}{2} \mathbb{1}-\varphi_{h}^{k}\right)+f\left(u_{h}^{k}\right) \tilde{\mathbf{e}}, \boldsymbol{\eta}-\boldsymbol{\varphi}_{h}^{k+1}\right)_{h}
\end{aligned}
$$


Here $(\eta, \chi)_{h}=\int_{\Omega_{h}} I_{h}(\eta \chi)$ denotes the discrete $L^{2}$ inner product over $\Omega_{h}$ with $I_{h}$ being the node-wise interpolant operator. The above variational inequality can be solved by setting, for $i=1, \ldots, M$ and $j=1, \ldots, 2 N$,

$$
\begin{aligned}
\left(\bar{\varphi}_{h}^{k+1}, \boldsymbol{\xi}_{i j}\right)_{h}:= & \left(\varphi_{h}^{k}, \boldsymbol{\xi}_{i j}\right)_{h}-\Delta t\left(T F_{X}\left(\varphi_{h}^{k}, \nabla \varphi_{h}^{k}\right), \nabla \boldsymbol{\xi}_{i j}\right)_{h} \\
& -\Delta t\left(T F_{\varphi}\left(\varphi_{h}^{k}, \nabla \varphi_{h}^{k}\right), \boldsymbol{\xi}_{i j}\right)_{h}-\frac{\Delta t}{\varepsilon}\left(\frac{1}{\varepsilon} T\left(\frac{1}{2} \mathbb{1}-\varphi_{h}^{k}\right)_{h}+T f\left(u_{h}^{k}\right) \tilde{\mathbf{e}}, \boldsymbol{\xi}_{i j}\right)_{h},
\end{aligned}
$$

where $\boldsymbol{\xi}_{i j}=\xi_{i} \mathbf{e}_{j}$, and then defining at each mesh point $\mathbf{x}_{j} \in \mathcal{N}_{h}$ the value of $\varphi_{h}^{k+1}\left(\mathbf{x}_{j}\right)$ to be the projection of $\bar{\varphi}_{h}^{k+1}\left(\mathbf{x}_{j}\right)$ onto the Gibbs simplex $\mathcal{G}$ (see for example [8] and [20] for the scalar case).

To discretize the equation for the solute $u$ we use an implicit Euler scheme in time and discretize the weak formulation of 2.6) and 2.7 in the following way. We seek $u_{h}^{k+1}$ such that for all $\chi \in S_{h}$,

$$
\frac{\gamma \varepsilon}{\Delta t}\left(u_{h}^{k+1}-u_{h}^{k}, \chi\right)_{h}+\left(D\left(\varphi_{h}^{k}\right) \nabla v_{h}^{k+1}, \nabla \chi\right)_{h}+\alpha \int_{\partial \Omega_{h}} I_{h}\left(D\left(\varphi_{h}^{k}\right)\left(u_{h}^{k+1}-1\right) \chi\right)=0,
$$

where

$$
v_{h}^{k+1}=u_{h}^{k+1}+\varepsilon f^{\prime}\left(u_{h}^{k+1}\right) \sum_{i=1}^{N} \varphi_{h, i}^{k} .
$$

For the case of thin specimens we couple (4.1) with the node-wise approximation of (2.9) such that for all nodes $j$,

$$
u_{i}^{k}= \begin{cases}1 & \forall i \text { such that } \varphi_{i, j}^{\bar{k}}>0 \text { for any } i \in\{N+1, \ldots, 2 N\} \text { and } 0 \leqslant \bar{k} \leqslant k \\ 0 & \text { otherwise. }\end{cases}
$$

For initial data $\varphi_{h}^{0}(\mathbf{x})$ and $u_{h}^{0}(\mathbf{x})$ we interpolate 2.12 .

\subsection{Sharp interface discretization}

In order to discretize the sharp interface model we consider a parametric formulation.

4.2.1 Parametric formulation. In the following we consider the situation of three interfaces $\Gamma_{i}$, $i=1,2,3$, that meet at a triple junction with prescribed angles and that evolve with velocity laws

$$
V_{i}=\kappa_{i}+\frac{4}{\pi} \mathcal{F}_{i}, \quad i=1,2,3 .
$$

If we use a parametrization $\mathbf{X}_{i}(p, t)=\left(x_{i}(p, t), y_{i}(p, t)\right)$ of $\Gamma_{i}(t)$, where $p$ is a spatial parameter and $t$ is time, equations (3.1) and 3.4) yield

$$
\begin{gathered}
\mathbf{X}_{i, t}=\frac{\mathbf{X}_{i, p p}}{\left|\mathbf{X}_{i, p}\right|^{2}}+\frac{4 \mathcal{F}_{i}}{\pi} \frac{\left(\mathbf{X}_{i, p}\right)^{\perp}}{\left|\mathbf{X}_{i, p}\right|}, \\
-\gamma \mathbf{X}_{i, t} \cdot\left(\mathbf{X}_{i, p}\right)^{\perp}[u]_{\Gamma_{i}}=\frac{\pi}{8}\left(\frac{u_{i, p}}{\left|\mathbf{X}_{i, p}\right|}\right)_{p},
\end{gathered}
$$

where

$$
\mathbf{X}_{i}:[0,1] \times[0, T] \rightarrow \mathbb{R}^{2}, \quad(p, t) \mapsto \mathbf{X}_{i}(p, t),
$$


and $\left(\alpha_{1}, \alpha_{2}\right)^{\perp}=\left(\alpha_{2},-\alpha_{1}\right)$ (compare [7]). To be precise let us mention that a solution of 4.5 ) leads to a parametrization which fulfills (3.1). But (3.1) only determines the normal velocity of $\mathbf{X}_{i}$, while the tangential velocity of $\mathbf{X}_{i}$ is determined by (4.5) and in principal other choices are possible (see [8]).

Denote the angle between $\Gamma_{1}$ and $\Gamma_{2}$ by $\theta_{1}$ and the angle between $\Gamma_{2}$ and $\Gamma_{3}$ by $\theta_{2}$. Then the triple point condition (3.8) may be written as

$$
\begin{aligned}
& \frac{\mathbf{X}_{1, p}}{\left|\mathbf{X}_{1, p}\right|} \cdot \frac{\mathbf{X}_{2, p}}{\left|\mathbf{X}_{2, p}\right|}=\cos \left(\theta_{1}\right), \\
& \frac{\mathbf{X}_{2, p}}{\left|\mathbf{X}_{2, p}\right|} \cdot \frac{\mathbf{X}_{3, p}}{\left|\mathbf{X}_{3, p}\right|}=\cos \left(\theta_{2}\right) .
\end{aligned}
$$

The balance law 3.10 ) for the concentration takes the form

$$
\sum_{i=1}^{3} \frac{u_{i, p}}{\left|\mathbf{X}_{i, p}\right|}=0
$$

We let $b(s)$ denote the domain boundary in terms of an arc length parameter $s$. Then the junction conditions at the physical boundary are given by

$$
\mathbf{X}_{i}(0, t)=b\left(s_{i}(t)\right), \quad u_{i}(0, t)=1, \quad i=1,2,3,
$$

for some $s_{i}(t)$, and

$$
\mathbf{X}_{i, p}(0, t) \cdot b^{\prime}\left(s_{i}(t)\right)=0,
$$

where' denotes differentiation with respect to $s$.

\subsection{Discretization of parametric formulation}

To discretize (4.5) and (4.6) we follow the ideas presented in [9] where the discrete approximation to $u$ is defined on a "background" fine, uniform mesh $\mathcal{M}$, with grid size $\tilde{h}$. In particular we let $u_{h}^{n}(\mathbf{x})=u_{h}^{n}\left((\mathbf{x})^{m}, t\right)$, for all $t \in[n \Delta t,(n+1) \Delta t)$ and all $(\mathbf{x})^{m} \in \mathcal{M}$, be a discrete approximation of the concentration $u$ such that for any $\mathbf{x} \in \Omega$ we define $(\mathbf{x})^{m}$ to be its nearest node on $\mathcal{M}$. Furthermore we define $\mathbf{X}_{i, j}^{n}=\mathbf{X}_{i}\left(s_{j}, t\right)$ and $u_{i, j}^{n}=u_{i}\left(s_{j}, t\right)$ for all $t \in[n \Delta t,(n+1) \Delta t), j=(0, \ldots, M)$, to be discrete approximations of $\mathbf{X}_{i}$ and $u_{i}$ respectively.

To solve 4.5 we adapt the techniques in [3, 7, 8] to obtain the following approximation of [4.5) for any grid point $j \in[1, M-1]$ :

$$
\begin{aligned}
\frac{1}{2}\left(\left(h_{j+1}\right)^{2}+\left(h_{j}\right)^{2}\right) & \dot{\mathbf{X}}_{i, j}=\left(\mathbf{X}_{i, j+1}-2 \mathbf{X}_{i, j}+\mathbf{X}_{i, j-1}\right) \\
+ & \frac{2 \mathcal{F}_{i, j}}{\pi}\left(h_{j+1}\left(\left(\mathbf{X}_{i, j+1}\right)^{\perp}-\left(\mathbf{X}_{i, j}\right)^{\perp}\right)+h_{j}\left(\left(\mathbf{X}_{i, j}\right)^{\perp}-\left(\mathbf{X}_{i, j-1}\right)^{\perp}\right)\right) .
\end{aligned}
$$

Here $h_{j}=\left|\mathbf{X}_{j}-\mathbf{X}_{j-1}\right|$, and $\mathcal{F}_{i, j}$ which is defined later is an approximation to 3.2 . For the boundary data $\mathbf{X}_{i, 0}$, it suffices to set

$$
\mathbf{X}_{i, 0}=2 B_{i}-\mathbf{X}_{i, 1} \quad \text { for } i=1,2,3
$$


where $B_{i}$ is the point on the boundary with minimum distance to $\mathbf{X}_{i, 1}$ (see [3] for more details). We approximate (4.7) and (4.8) by

$$
\begin{aligned}
& \frac{D_{+} \mathbf{X}_{1, M-1}}{\left|D_{+} \mathbf{X}_{1, M-1}\right|} \cdot \frac{D_{+} \mathbf{X}_{2, M-1}}{\left|D_{+} \mathbf{X}_{2, M-1}\right|}=\cos \left(\theta_{1}\right) \\
& \frac{D_{+} \mathbf{X}_{2, M-1}}{\left|D_{+} \mathbf{X}_{2, M-1}\right|} \cdot \frac{D_{+} \mathbf{X}_{3, M-1}}{\left|D_{+} \mathbf{X}_{3, M-1}\right|}=\cos \left(\theta_{2}\right)
\end{aligned}
$$

where $D_{+} \mathbf{X}_{i, j}=\left(\mathbf{X}_{i, j+1}-\mathbf{X}_{i, j}\right) / h$. We see from [3] that by simple geometric formulas it is possible to uniquely determine $\mathbf{X}_{i, M}$ from $\mathbf{X}_{i, M-1}$ for $i=1,2,3$.

We approximate 3.2 by

$$
\mathcal{F}_{i, j}= \begin{cases}0 & \text { if } i, j \in[1, N], \\ 0 & \text { if } i, j \in[N+1,2 N], \\ {\left[f\left(u_{h}^{n}\right)\right] \mathbf{X}_{i, j}} & \text { if } i \in[1, N], j \in[N+1,2 N], j \neq i+N,\end{cases}
$$

where

$$
\left[f\left(u_{h}^{n}\right)\right] \mathbf{X}_{i, j}:=f\left(u_{h}^{n}\left(\left(\mathbf{X}_{i, j}+\delta v_{i, j}\right)^{m}\right)\right)-f\left(u_{h}^{n}\left(\left(\mathbf{X}_{i, j}-\delta v_{i, j}\right)^{m}\right)\right)
$$

and $v_{i, j}=\left(\mathbf{X}_{i, j}\right)_{s}^{\perp} /\left|\left(\mathbf{X}_{i, j}\right)_{s}\right|$ with $\left(\mathbf{X}_{i, j}\right)_{s}^{\perp}=\left(y_{i, j+1}-y_{i, j-1}, x_{i, j-1}-x_{i, j+1}\right)$. We choose $\delta$ to be $\mathcal{O}(\tilde{h})$ so that we test the values of $u_{h}$ on either side of the interface. We approximate 4.6 for any $j \in[1, M-1]$ by

$$
-\frac{\gamma\left(\mathbf{X}_{i, j}^{n+1}-\mathbf{X}_{i, j}^{n}\right)}{2 \Delta t} \cdot\left(\left(\mathbf{X}_{i, j+1}^{n+1}\right)^{\perp}-\left(\mathbf{X}_{i, j-1}^{n+1}\right)^{\perp}\right)\left[u_{h}^{n}\right]_{\mathbf{X}_{i, j}^{n}}=\frac{\pi}{8}\left(\frac{D_{+} u_{i, j}^{n+1}}{h_{j+1}^{n}}-\frac{D_{+} u_{i, j-1}^{n+1}}{h_{j}^{n}}\right),
$$

where $\left[u_{h}^{n}\right]_{\mathbf{X}_{i, j}^{n}}:=u_{h}^{n}\left(\left(\mathbf{X}_{i, j}^{n}+\delta v_{i, j}^{n}\right)^{m}\right)-u_{h}^{n}\left(\left(\mathbf{X}_{i, j}^{n}-\delta v_{i, j}^{n}\right)^{m}\right)$. To approximate the boundary data (3.5) and 3.6 we set

$$
\frac{D_{+} u_{i, 0}^{n+1}}{\left|D_{+} \mathbf{X}_{i, 0}^{n+1}\right|}=\alpha\left(1-u_{i, 0}^{n+1}\right) \quad \text { and } \quad u_{i, 0}^{n+1}=1
$$

respectively. Lastly we require that the concentration of the solute is continuous across the triple junction 3.9 , i.e. $u_{i, M}^{n+1}=u_{M}^{n+1}$ for $i=1,2,3$ and that a discrete version of the force balance law 3.10 holds:

$$
\sum_{i} \frac{D_{+} u_{i, M-1}^{n+1}}{\left|D_{+} \mathbf{X}_{i, M-1}^{n+1}\right|}=0
$$

We update $u_{h}^{n+1}(\mathbf{x})$ for all $\mathbf{x} \in \mathcal{M}$ in the following way:

$$
u_{h}^{n+1}(\mathbf{x})= \begin{cases}\frac{1}{2}\left(u_{i, j}^{n+1}+u_{i, j+1}^{n+1}\right) & \text { if } \mathbf{x} \in S_{i, j}, j=0, \ldots, M-1, i=1,2,3, \\ u_{h}^{n}(\mathbf{x}) & \text { otherwise }\end{cases}
$$

where $S_{i, j}$ denotes the region enclosed by the four lines that join the points $\left(\mathbf{X}_{i, j}^{n}\right)^{m}$ and $\left(\mathbf{X}_{i, j}^{n+1}\right)^{m}$, $\left(\mathbf{X}_{i, j+1}^{n}\right)^{m}$ and $\left(\mathbf{X}_{i, j+1}^{n+1}\right)^{m},\left(\mathbf{X}_{i, j}^{n}\right)^{m}$ and $\left(\mathbf{X}_{i, j+1}^{n}\right)^{m}$ and $\left(\mathbf{X}_{i, j}^{n+1}\right)^{m}$ and $\left(\mathbf{X}_{i, j+1}^{n+1}\right)^{m} ;$ see Figure 3 


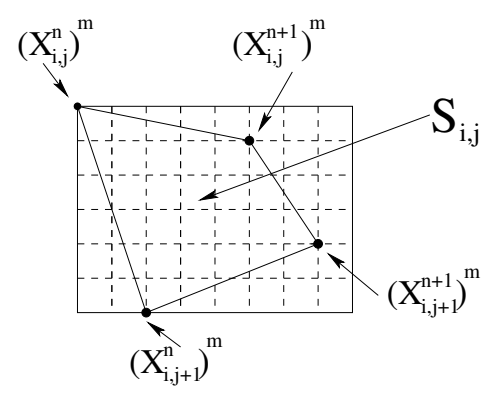

FIG. 3. Updating $u_{h}^{n}$.

\section{Numerical results}

In this section we present phase field and sharp interface simulations using the numerical discretizations derived in Section 4. For the phase field simulations we used a Gauss-Seidel algorithm to solve 4.2 and for the sharp interface simulations we used a fourth-order Runge-Kutta algorithm (with the forcing term $\mathcal{F}_{i, j}$ taken at the old time level) to solve (4.12) and a Gauss-Seidel algorithm to solve (4.13). We display the values of the parameters $\varepsilon, h$, etc. that were used in each simulation in Tables 1 and 2 .

TABLE 1

Parameters used in phase field simulations

\begin{tabular}{|c|c|c|c|}
\hline Figure & $h$ & $\Delta t$ & $\varepsilon$ \\
\hline 4 & 0.005 & $h^{2} / 40$ & 0.025 \\
\hline 5 & 0.03 & $h^{2} / 40$ & 0.16 \\
\hline 6 and 7 & 0.01 & $h^{2} / 100$ & 0.05 \\
\hline 8 and 9 & 0.025 & $h^{2} / 40$ & 0.0125 \\
\hline
\end{tabular}

TABLE 2

Parameters used in sharp interface simulations

\begin{tabular}{|c|c|c|c|c|}
\hline Figure & $N$ & $h$ & $\Delta t$ & $\tilde{h}$ \\
\hline 10 and 11 & 100 & 0.02 & $h^{2} / 40$ & 0.005 \\
\hline 12 and 13 & 200 & 0.005 & $h^{2} / 80$ & 0.0025 \\
\hline 17 & 1200 & 0.1 & $h^{2} / 40$ & 0.3 \\
\hline
\end{tabular}

For all of the phase field simulations we took

$$
F(\varphi, \nabla \varphi)=\frac{1}{2} \sum_{i=1}^{N}\left|\nabla\left(\varphi_{i}+\varphi_{i+N}\right)\right|^{2} .
$$


The simulations correspond to two types of experimental set-ups, which henceforth we will refer to as thin film and thick film. In the thin film set-up the domain $\Omega \subset \mathbb{R}^{2}$ corresponds to a horizontal cross section through the film and the concentration of solute which is assumed to be constant through the film satisfies 2.9p. In the thick film set-up the domain $\Omega \subset \mathbb{R}^{2}$ corresponds to a vertical cross section through the film with the concentration satisfying the diffusion equation (2.6) or (3.4) with $\gamma=0.25$. We do not display any plots of the concentration in the thin film simulations since it only takes the values zero or one, instead regions of positive concentration are denoted by hashed areas. For the thick film simulations we display plots of the grain boundaries evolving in time together with plots of the concentration at the final evolution times.

The first two simulations, Figures 4 and 5 are two thin film phase field computations that reproduce numerical simulations presented in [9] and [11] using our new model. The remaining figures display a combination of phase field and sharp interface simulations that show the evolution of three grain boundaries meeting at triple junctions.

\subsection{Phase field simulations}

5.1.1 Thin film. The first two thin film phase field simulations (Figures 4 and 5 ) reproduce numerical simulations presented in [9, 11], where an initially straight grain boundary that separates two grain orientations evolves in time to form

(i) a stationary solution of two circular arcs with radius 1 (Figure 4).

(ii) a double seam, where the grain boundary doubles back on itself (Figure 5).

When we compared the solutions in Figures 4 and 5 with solutions using the phase field model presented in [9] the grain boundary evolution for the two models was graphically indistinguishable.

The remaining two thin film simulations show the evolution of three initially straight grain boundaries in a circular domain. The three boundaries meet at a triple junction and are separated by angles of $2 \pi / 3$. In Figure 6 all boundaries move in an anti-clockwise direction while in Figure 7 two boundaries move in anti-clockwise directions while the third moves in a clockwise direction.

In the symmetric case of Figure 6 although the grain boundaries migrate the triple junction remains fixed, while in the non-symmetric case of Figure 7 the triple junction also migrates.

5.1.2 Thick film. We display two thick film phase field figures; the first (Figure 8) shows the evolution of three grain boundaries meeting at a triple junction, while the second (Figure 9) shows the concentration in this simulation at the final time $t=0.04$.

\subsection{Sharp interface simulations}

The sharp interface simulations displayed in Figures $10+13$ reproduce the phase field simulations (Figures 6 9). In each subplot the initial positions of the grain boundaries are represented by dashed lines, while the positions of the grain boundaries after time $t>0$ (displayed at the top of the subplot) are represented by solid lines.

\subsection{Comparison of phase field and sharp interface simulations}

In Figures $14 \sqrt{16}$ we display comparisons between phase field and sharp interface solutions in which a " $*$ " is plotted at every 20th node of the sharp interface discretization. Figure 14 displays 
H. GARCKE \& V. STYLES
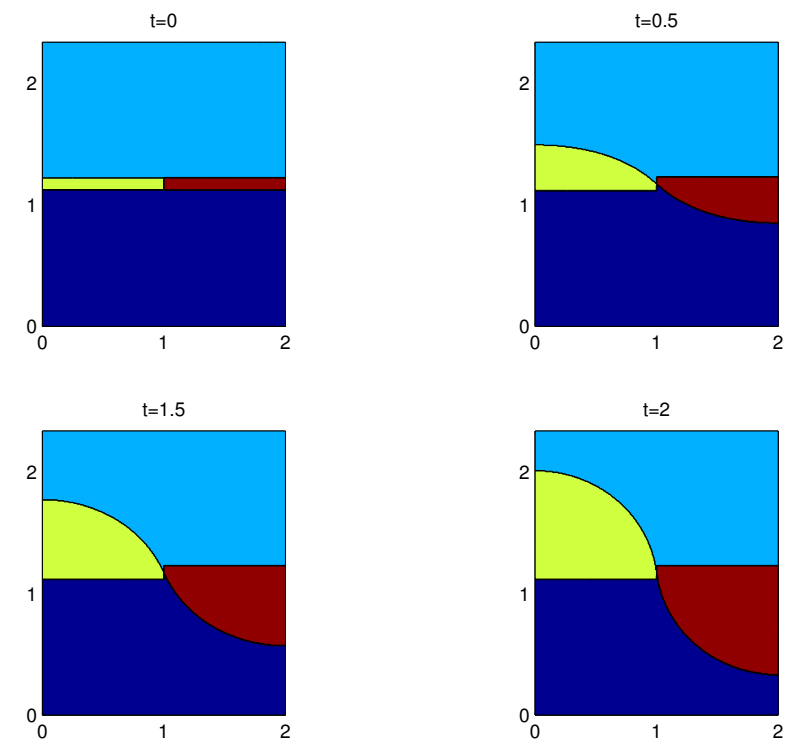

FIG. 4. Thin film phase field solutions solution.
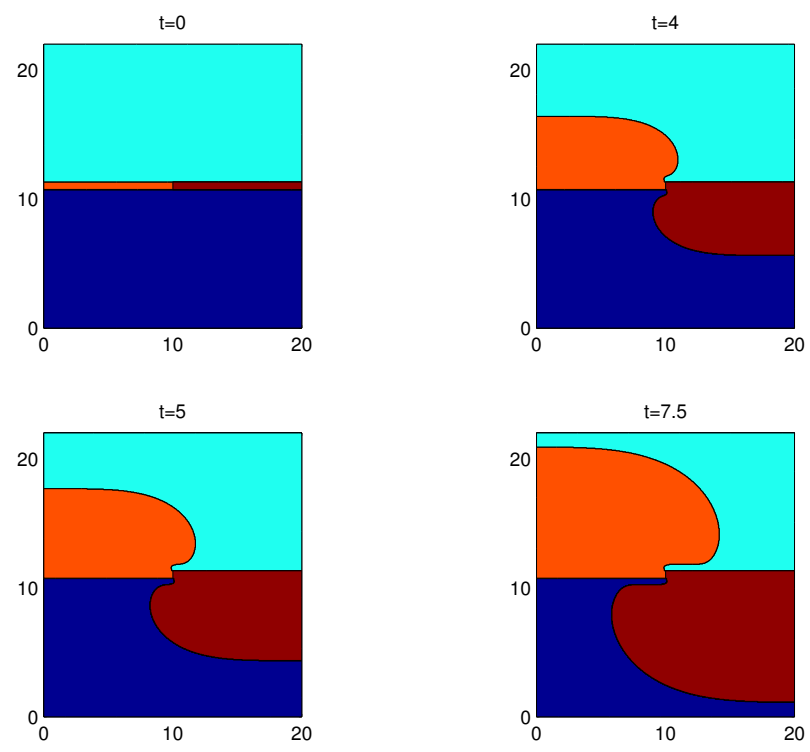

FIG. 5. Thin film phase field solutions. 

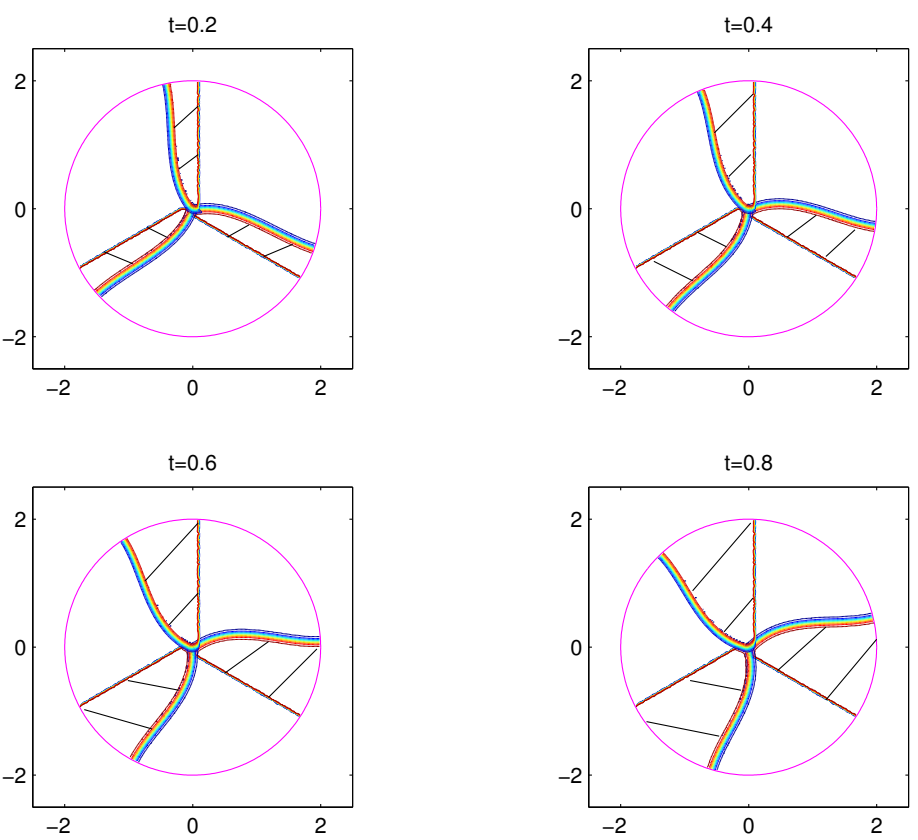

FIG. 6. Phase field solutions of a symmetric triple junction.
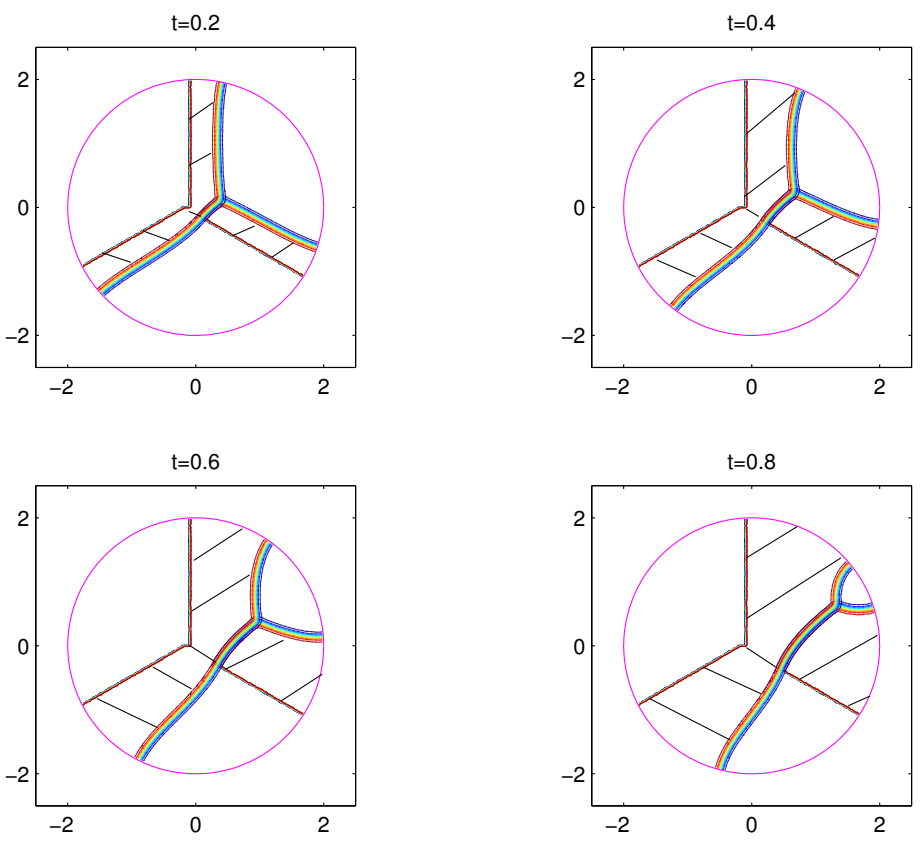

FIG. 7. Phase field solutions of a nonsymmetric triple junction. 

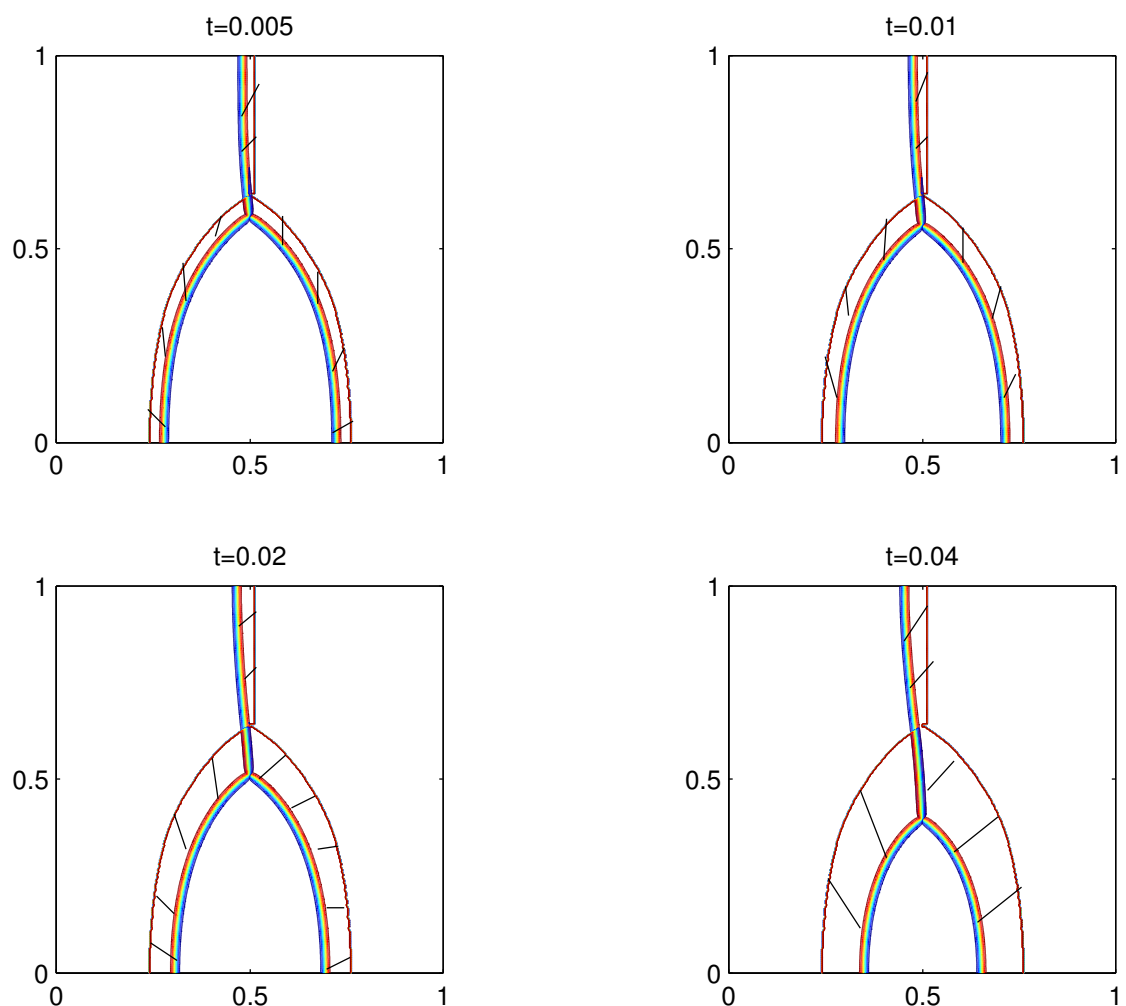

FIG. 8. Phase field thick film solutions

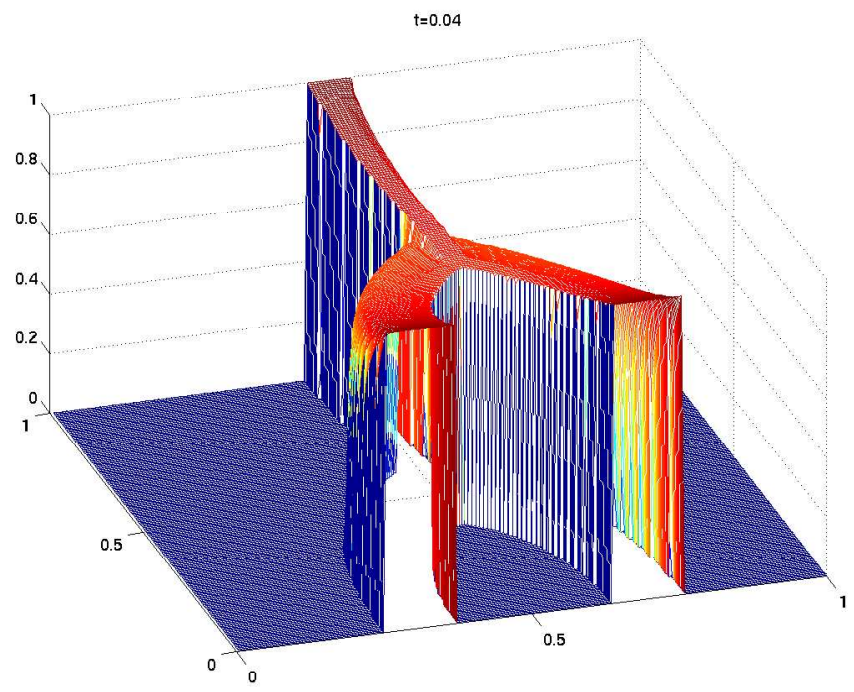

FIG. 9. Concentration of solute in phase field thick film simulation. 

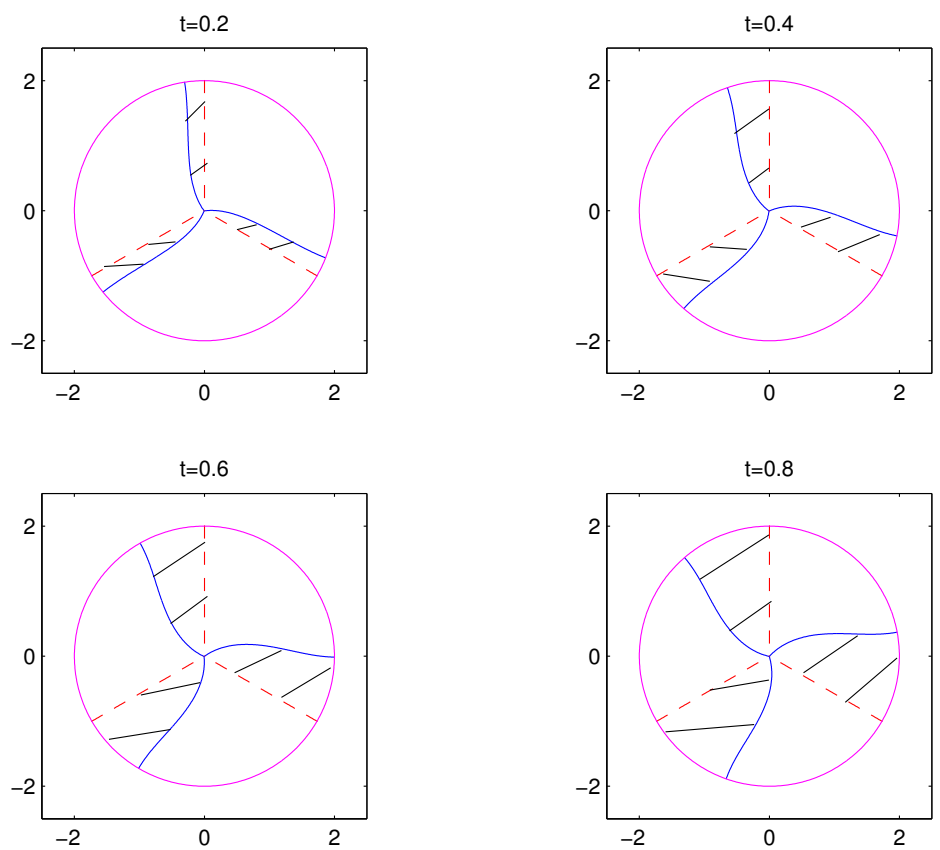

FIG. 10. Sharp interface solutions of a symmetric triple junction.
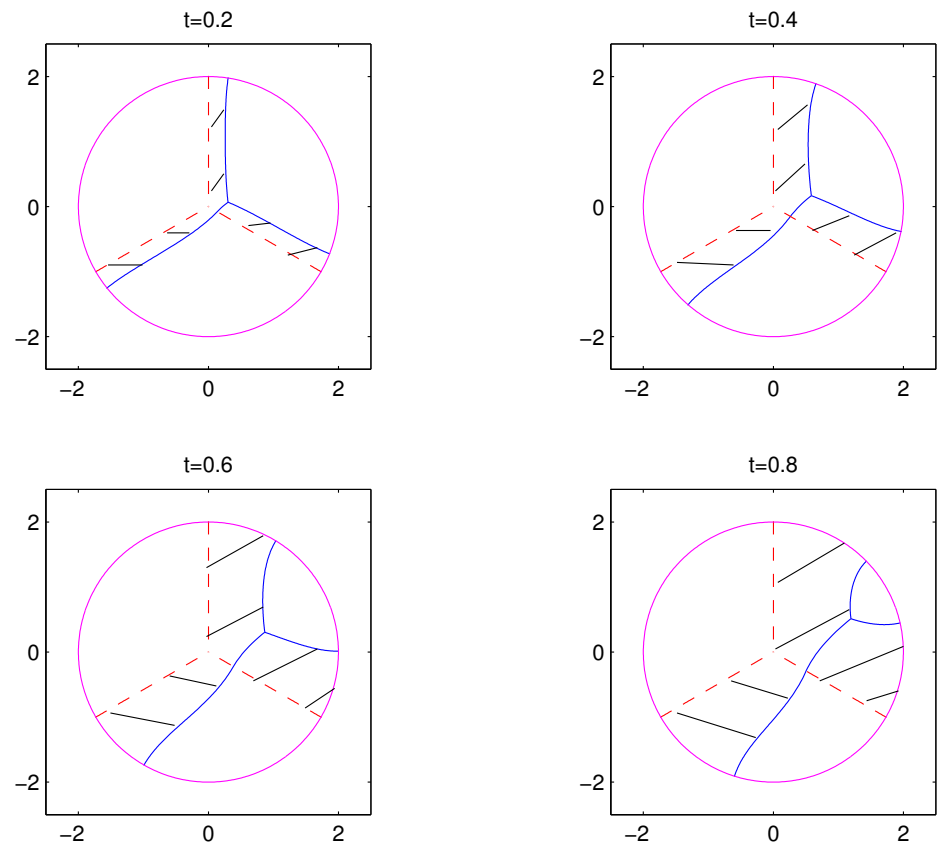

FIG. 11. Sharp interface solutions of a nonsymmetric triple junction. 

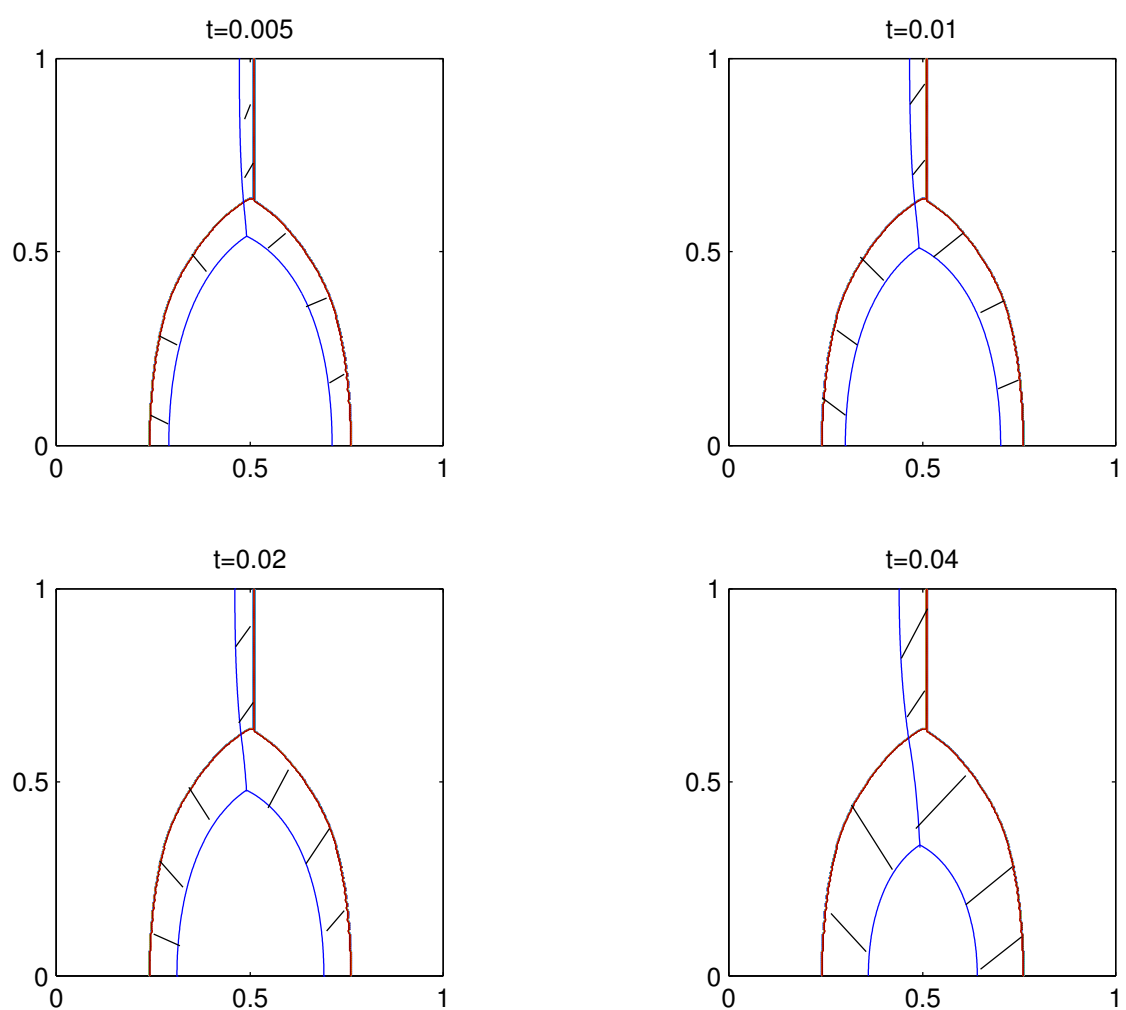

FIG. 12. Sharp interface thick film solutions.

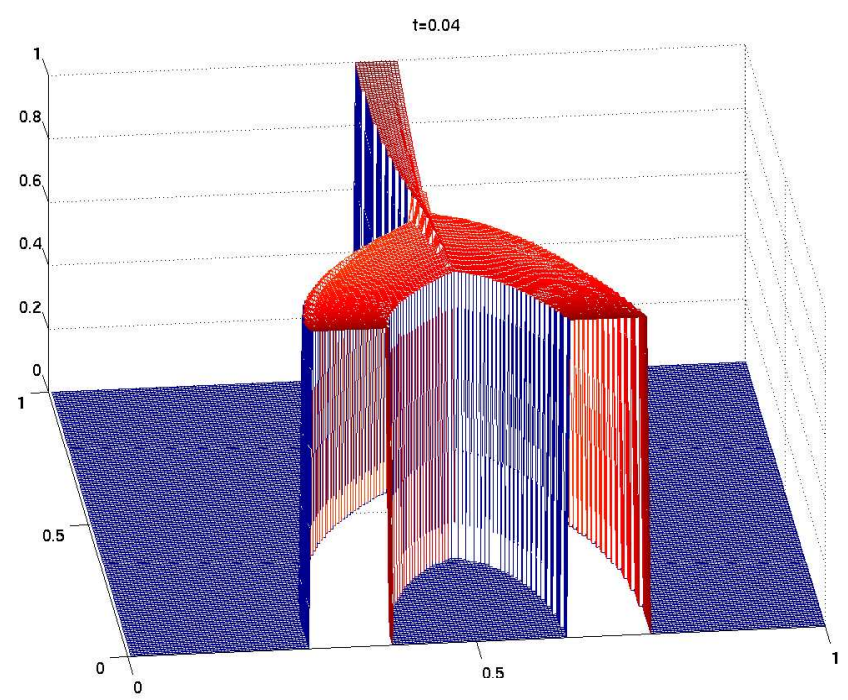

FIG. 13. Concentration of solute in above sharp interface thick film simulation. 


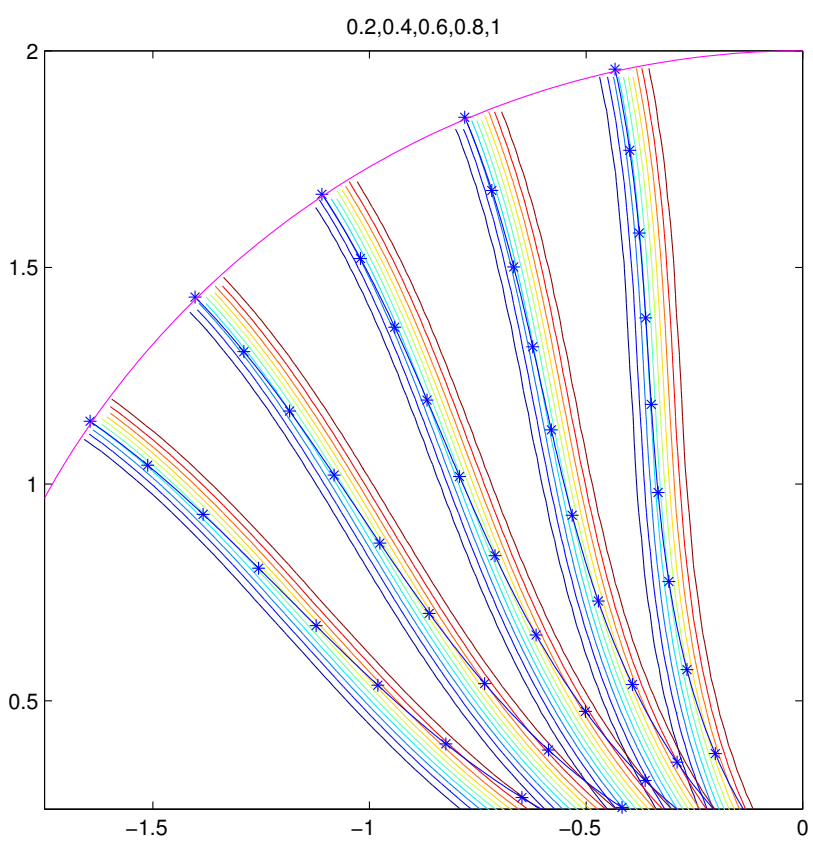

FIG. 14. Comparison of thin film phase field and sharp interface solutions.
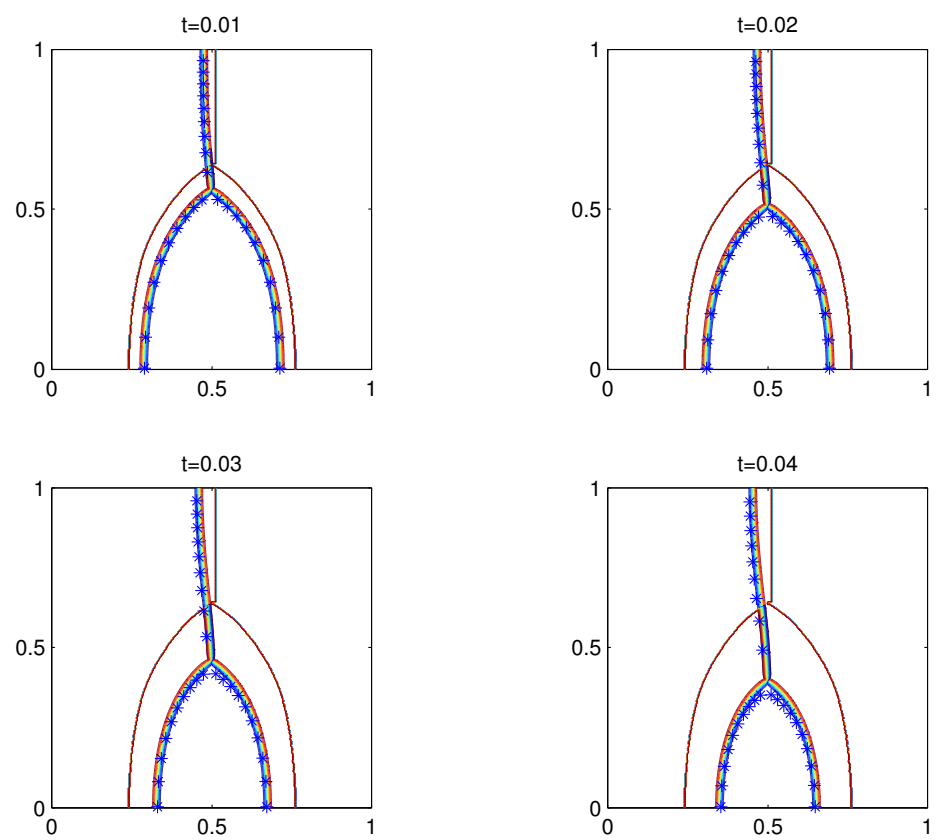

FIG. 15. Comparison of thick film sharp interface and phase field interfacial positions. 

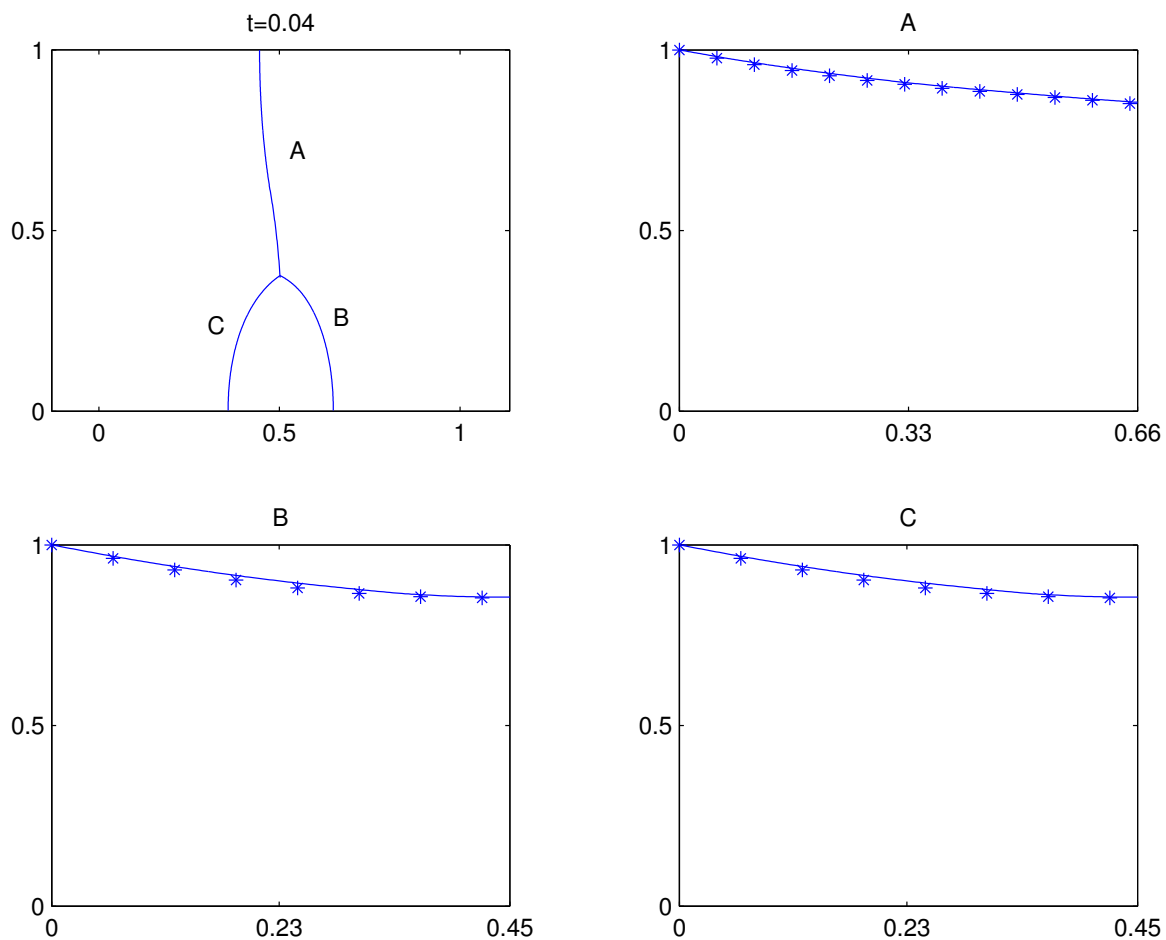

FIG. 16. Comparison of thick film sharp interface and phase field concentration solutions.
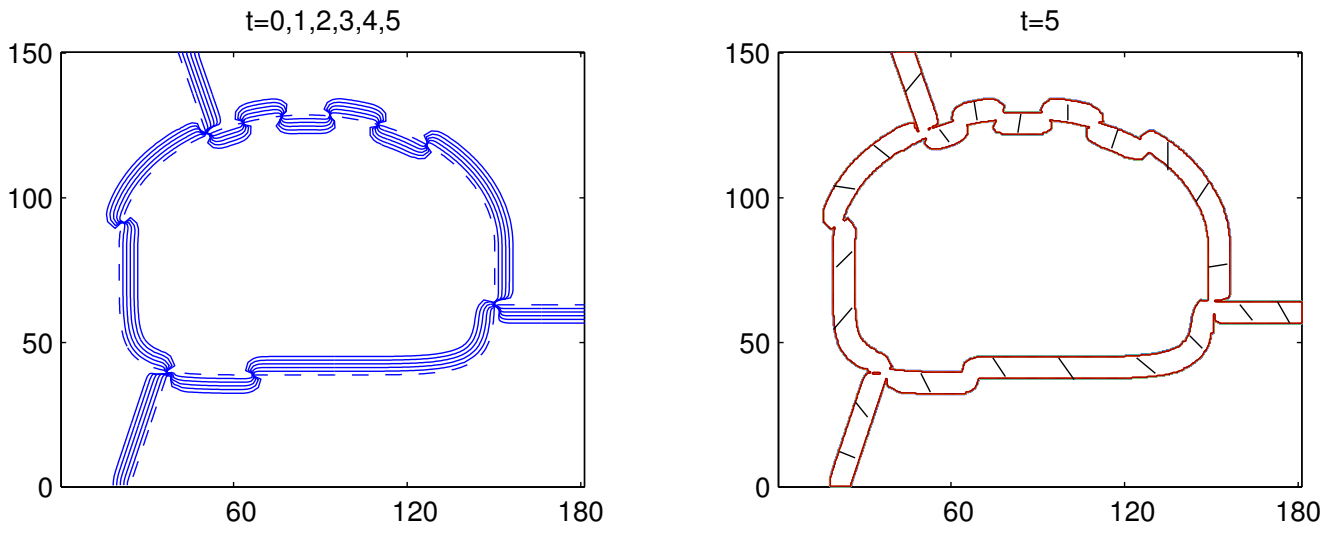

FIG. 17. Thin film sharp interface solutions. 
comparisons between the thin film solutions shown in Figures 6 and 10 , while Figure 15 displays comparisons of the interfacial positions in the thick film simulations shown in Figures 8 and 12 . In order to compare the concentration $u_{h}(\mathbf{x}, t)$ in the phase field model with the concentrations $u_{i}(s, t)$ in the sharp interface model we define $\tilde{u}_{i}(s, t)=u_{h}\left(\tilde{\Gamma}_{i}(s, t), t\right)$, where $\tilde{\Gamma}_{i}(\cdot, t)$ is a parametrization of $\tilde{\Gamma}_{i}(t)=\left\{\mathbf{x} \in \Omega: \varphi_{i}(\mathbf{x}, t)=0.5\right\}$ and $s$ is the arc length of $\tilde{\Gamma}_{i}(t)$ measured from the boundary. In Figure 16 we show $\tilde{u}_{i}$ (bold line) and $u_{i}$ ('*') plotted against arc length.

\subsection{A more complicated structure}

We conclude our simulations with Figure 17 in which we attempt to simulate a situation that appears in real microstructure formation (see [17, Figure 3, p. 249]). The simulation is obtained by using the sharp interface thin film model with the lefthand plot showing six grain boundaries evolving in time from $t=0$ (dashed lines) to $t=5$ (solid lines), while the righthand plot shows the alloyed region at time $t=5$.

\section{Acknowledgements}

This work was supported by the RTN Programme Fronts-Singularities, HPRN-CT-2002-00274.

\section{REFERENCES}

1. Blowey, J. F. \& Elliott, C. M. Curvature dependent phase boundary motion and parabolic double obstacle problems. Degenerate Diffusions, W.-M. Ni et al. (eds.), IMA Vol. Math. Anal. 47, Springer, New York (1993), 19-60. Zbl 0794.35092 MR 1246337

2. BRONSARD, L. \& REITICH, F. On three-phase boundary motion and the singular limit of a vector-valued Ginzburg-Landau equation. Arch. Rat. Mech. Anal. 124 (1993), 355-379. Zbl 0785.76085 MR 1240580

3. BRonsARD, L. \& Wetton, B. A numerical method for tracking curve networks moving with curvature motion. J. Comput. Phys. 120 (1995), 66-87. Zbl 0838.65087 MR 1345029

4. Cahn, J. W., Fife, P., \& Penrose, O. A phase-field model for diffusion-induced grain-boundary motion. Acta Mater. 45 (1997), 4397-4413.

5. Ciarlet, P. G. The Finite Element Method for Elliptic Problems. North-Holland, Amsterdam (1978). Zbl 0383.65058 MR 0520174

6. Deckelnick, K. \& Elliott, C. M. An existence and uniqueness result for a phase-field model of diffusion-induced grain-boundary motion. Proc. Roy. Soc. Edinburgh Ser A. 131 (2001), 1323-1344. Zbl 0989.35006 MR 1869638

7. Deckelnick, K., Elliott, C. M., \& Styles, V. Numerical diffusion induced grain boundary motion. Interfaces Free Bound. 3 (2001) 393-414. Zbl $0991.35095 \mid$ MR 1869586

8. Elliott, C. M. Approximation of curvature dependent interface motion. The State of the Art in Numerical Analysis, IMA Conf. Ser. 63, Clarendon Press, Oxford (1997), 407-440. Zbl 0881.65131 MR 1628355

9. Elliott, C. M. \& Styles, V. Computations of bi-directional grain boundary dynamics in thin films. J. Comput. Phys. 187 (2003), 524-543. Zbl 1020.82006 MR 1980269

10. Fife, P., CAhn, J. W., \& Elliott, C. M. A free boundary model for diffusion-induced grain-boundary motion. Interfaces Free Bound. 3 (2001), 291-336. Zbl 0986.35136 MR 1843589

11. Fife, P. \& WANG, X.-P. Chemically induced grain boundary dynamics, forced motion by curvature, and the appearance of double seams. Eur. J. Appl. Math. 13 (2002), 25-52. Zbl pre01796088 MR 1895306 
12. Garcke, H., Nestler, B., \& Stoth, B. On anisotropic order parameter models for multi-phase systems and their sharp interface limits. Phys. D 115 (1998), 87-108. Zbl 0936.82010 MR 1616772

13. Garcke, H., Nestler, B., \& StOTh, B. Anisotropy in multi-phase systems: a phase field approach. Interfaces Free Bound. 1 (1999), 175-198. Zbl 0959.35169 MR 1867130

14. Garcke, H., Nestler, B., \& Stoth, B. A multiphase field concept: Numerical simulations of moving phase boundaries and multiple junctions. SIAM J. Appl. Math. 60 (1999), 295-315. Zbl 0942.35095 MR 1740846

15. GARCKe, H. \& Novick-Cohen, A. A singular limit for a system of degenerate Cahn-Hilliard equations. Adv. Differential Equations 5 (2000), 401-434. Zbl 0988.35019 MR 1750107

16. Gurtin, M. E. Thermodynamics of Evolving Phase Boundaries in the Plane. Clarendon Press, Oxford (1993). Zbl 0787.73004 MR 1402243

17. HANDWERKER, C. Diffusion-induced grain boundary migration in thin films. Diffusion Phenomena in Thin Films and Microelectronic Materials, D. Gupta and P. S. Ho (eds.), Noyes, Park Ridge, NJ (1988), 245-322.

18. Herring, C. Surface tension as a motivation for sintering. The Physics of Powder Metallurgy (Chapter 8), W. E. Kingston (ed.), McGraw-Hill, New York (1951).

19. ModicA, L. The gradient theory of phase transitions and the minmal interface criterion. Arch. Rat. Mech. Anal. 98 (1987), 123-142. Zbl 0616.76004 MR 0866718

20. Nochetto, R. H., Paolini, M., \& Verdi, C. A dynamic mesh method for curvature dependent evolving interface. J. Comput. Phys. 123 (1996), 296-310. Zbl $0851.65067 \mid$ MR 1372375

21. Rubinstein, J., Sternberg, P., \& Keller, J. B. Fast reaction, slow diffusion and curve shortnening. SIAM J. Appl. Math. 49 (1989), 116-133. Zbl 0701.35012 MR 0978829

22. Purdy, G. R. Transformations involving interfacial diffusion. Materials Science and Technology, A Comprehensive Treatment, R. W. Cahn et al. (eds.), VCH Verlag, Weinheim (1990), 305-338.

23. Sutton, A. P. \& BAlluff, R. W. Interfaces in Crystalline Materials, Oxford Univ. Press (1995). 